\title{
Sports Injury Risks and Opportunity Costs: The Conspicuous Landscape of Handball among Tertiary Teachers' Colleges in Zimbabwe
}

\author{
Prince Chimonero, PhD \\ Great Zimbabwe University \\ Corresponding Mail: pchimonero@gzu.ac.zw
}

\begin{abstract}
This study explored injury risks associated with sport participation among handball players in Zimbabwean Tertiary Institutions between 2016 and 2019. The study employed a descriptive, prospective cohort design anchored on quantitative methodology and informed by Positivism Philosophy. The population comprised technocrats (coaches, fitness trainers, physiotherapists, psychologists and players) from selected Zimbabwe Teachers' Colleges Sports Association handball teams. Stratified random sampling was used to select the respondents. Questionnaire was used as data collection tool and IBM SPSS Statistic Version 23 was used for data analysis. Findings revealed critical knowledge-service gaps on sports medicine professionals regarding lack of players' informational sources on pre-participation medical health-checks as evidence-based practices for addressing activity limitations and risks upon return-to-sport. Ego-oriented administration characteristic of pain-injury paradox environment prevailed with pre-mature ending of players' rehabilitation routes. Well formulated guideline-inclined preventive injury risk management protocols resonating player-centered medicine approaches that could effectively abate epidemiologic opportunity injury risks and costs were non-existent. Furthermore, regularized in-service professional development clinics lacked. Players' objective injury records, profiles and pre-participation medical examination health-checks should be considered as critical informational sources for fully resolving physiological defects prior to return-to competitive contexts. Formulation of guideline-inclined preventive injury risk management protocols entrenching player-centered medicine practices could effectively trim down epidemiologic opportunity injury risks and costs and improve the critical base for players' odds of participation. Regularized inservice professional development trainings for sports medicine professionals through symposiums should inexorably be an esteemed epitomic pedestal delved towards improving their expert base levels in view of contemporary preventive and injury risk management dynamics in sports.
\end{abstract}

Keywords: Sports injury, collegiate handball, risk, Teachers Colleges, Sports Association

\section{Introduction}

Globally, people participate in sport for health and recreational reasons (Atchison et al., 2020). A minefield of literature substantiating public health awareness and motivational grounds for participating in sport exists (Dore et al., 2019; Shahri et al., 2019; Nemcek, 2017). In as much as this conveys noteworthy chain of contentment in elite professional and recreational sport (Berber \& Mollaogullan, 2020), injury threats, nevertheless, have become an inexorable trait (Battagia, Kerr \& Tamminen, 2021; Pierpoint \& Collins, 2021; Sanderson, 2021). Thus far, the issue of injury risk control in sporting populations has become a major contemporary concern for Sports Federations and Health Service providers (Gouttebarge et al., 2021, Bahr et al., 2020) in order to create safe play settings for players. Although enjoyment is a derivative of sports satisfaction, handball players often risk injury sustenance during participation. In spite of this, the nature of sports significantly determines the quantity of injury risks and mechanisms on players. While a myriad of confounding variables have been found to set unpalatable tendencies during players' participation, less attention seem to be given as to how their retrogressive impact could be abated. This manuscript, therefore, explored injury risks 
associated with participation among handball players in Zimbabwean Tertiary Institutions. It further sought to establish sports medicine professionals' conversance levels regarding sports injury risks and prevention in training and competitive settings.

This study adopted the precincts of the Protection Motivation Theory (Majewski, 2018) which provides potential restorative grounds in sports injury risks and management locales. The model allows for sports medicine professionals and coaches to work within pre-defined guideline-inclined parameters on injury risk preventive protocols. Further, the model practically provides curative frameworks and hoperestoration approaches that permit recuperating player's self-authorship to emotionally attest them to prescribed training protocols that re-orients musculoskeletal deficits (Shen et al., 2019). The PMT provides an operational framework on the fundamentals of rehabilitation, risk prevention and 'player centered care' concept. The model offered a road map to this study's nature and methodology (purpose, data analysis procedures, right through to research findings) in order to provide practical solutions that help reduce sports injury risk trends. From it, sports medicine professionals might derive and appropriately design preventive and restorative-oriented guidelines and approaches dealing with injured players, risk identification, risk reduction and prediction based on inter-disciplinary team work. This placed the researcher at a vantage point to predict and arrive at informed decisions that environ safe playing zones. Moreover, the model provided motivational environments for players to increase their adherence rates to prescribed rehabilitation regimes for their early come back to competitive contexts in view of their proximity to player-centered care.

\section{Review of Related Literature}

The concepts of "sports injury" and "risk control" have been underpinned by a biomedical doctrine of scientific objectivity (Bahr et al., 2001). Heterogeneous definitions have been proffered to explain the concepts of "injury" and "risk" (Fuller et al., 2006) have explored injury as any physical complaint a player sustains that results from match or training, regardless of medical attention or time loss and subsequently incapacitates player's full involvement in prospective trainings. In respect to their classification, Fuller et al. (2006) further expand traumatic injuries as having an identifiable onset while overuse injuries are a result of micro- trauma with no identifiable onset. Thus, injuries find their pathways within the outline of player-triggered and environmentally-triggered settings. Risk has been further explored as the likelihood of a phenomenon impacting on the objectives of an organization or institute (Australian Standard, 2004). Equally translated into sport context, the extent of tissue damage comes in handy (Baar, 2017; Trompeter et al., 2017; Grinden et al., 2017; Herman et al., 2017; Longo et al., 2017) as a result of biomechanical external and internal dynamic risk variables. Consequently, the concept of injury risk enmeshes high risks as a key concept.

High risk in any sport requires one's acceptance of the possibility of severe injury or death as an inherent part of the activity (Breivik et al., 1994) while physical risk is a defining feature (Palmer, 2002). Contemporary concepts of "sports injury risk control" have their pathways entrenched in biomedical and mechanist model of health (Hulme and Finch, 2015). To date, the hypothesis regarding force exposure overwhelming an organism's physiological injury threshold still resonates as the cornerstone of injury control (D'Hooghe et al., 2018; Longo et al., 2017; Hickey et al., 2017). For instance, load tolerance and load intensity rationalization during biomechanical maximal and sub-maximal workouts in relation to individual's aerobic training limitations are typical force production activities (Hughes, 2018; Landreau et al., 2018; Musahil et al., 2017).

\section{Underpinnings of Sports Injury Rehabilitations}

Individual athlete rehabilitation plan entails an assessment of the player's specific injury and more universal functional properties, alongside the practical knowledge on injury and treatment consequences (Frontera, 2003). Researchers (Pabian et al., 2017) suggest that accurate diagnoses of actual tissue damage come first, followed by injury presentation or the clinical symptoms multipart identification, injury type, the physiological and biomechanical factors associated with its occurrence. Ultimately, the acute method of treatment needs to be time-honored with a thorough acquaintance of the local or universal effects of the chosen modality (for example, immobilization, surgery, physical therapy) (Speerin et al., 2014). This becomes the launch base from which to ascertain appropriate rehabilitation strategies that addresses the full functional capacity of the athlete through a collaborative care-team of sports medicine professionals with appropriate skill 
sets (Fordsdyke, 2021; Shortway et al., 2017; Rahman, 2017; Clement et al., 2015; Broglie et al., 2014). Thus, rehabilitation as a process, should serve to fully re-enact the player void of physiological incapacities upon return-to-sport contexts through appropriately designed restorative-oriented training approaches.

\section{The Basis of Musculoskeletal Injury}

Musculoskeletal injuries contribute greater than $70 \%$ of time loss from active participation in sport. Besides, soft tissue injuries (tendon, ligament and muscle distressors) are characteristic features of sport participation at all levels (Baar, 2017) which determine the form of rehabilitation treatment to be prescribed. Research has demonstrated that specific tissues respond differently to the stress and strain associated with trauma. Injury occurrences often result when forces applied to a given anatomical structure exceed the physiological tolerance or reparative capabilities of the concerned structure (Frontera, 2003). As a gross step in injury assessment, injury types can be micro traumatic or macro traumatic in nature (Herring and Kibler, 1998). Macro trauma entails acute, extreme forces that overwhelm the tensile/structural properties of a given tissue due to acute deformation of previous structure. Besides, micro traumas appear in smaller forces with lesser degrees of structural disruption often at a rate or severity that exceeds the tissue's ability to enact appropriate repair or maintenance. This could weaken the structural properties of the affected tissue, adaptations of function in adjacent or compensatory structures, pain, impaired performance and; potentially, tissue failure (Frontera, 2003). Thus, the potency of colliding forces during maximal or sub-maximal pursuits critically determines injury severity. The greater the speed of impact, the greater the damage experienced on the limb (major injury). The lesser the speed of impact, the lesser the damage incurred on the site (minor injury). Consequently, management of injury type greatly varies together with their transitional levels of recovery during rehabilitation and return-to-sport.

Concussion is a widespread sports injury that emanates from short-lived decrements of neurocognitive function (Herman et al., 2017) which can lead to neuromuscular control impairments in gait oriented tasks such as obstacle clearance, gait initiation and gait termination (Chiu et al., 2016). These can be observed in symptomatic recuperation and return-to active participation (Martini et al.,
2011); hence is more likely to be accentuated during maximal explosive bouts. Players with poor neuromuscular control are believed to be more prone to musculoskeletal injuries during such high energy demanding workouts (Herman et al., 2017). Abnormal movement patterns in lower body appendages may result in muscle imbalances such as excessive loads on frontal plane motion at the knee and poor trunk control. Eventually, athletes become vulnerable to $\mathrm{ACL}$ injury and a host of multiple injuries (Grinden et al., 2017; Kam Mong Mok, 2015). Besides, athletes also risk developing back pain when engaged in high loaded workouts which pre-disposes them to mechanical overload on the body's musculotendinous units (Trompeter, Fett and Platen, 2017). Hence high bodily biomechanical coordinative exploitations during handball deceitful side cutting movements should proficiently be executed in order to eliminate erroneous causes of injury.

At a collegiate level, Lynall et al.'s (2016) study revealed high susceptibility levels (1.9 times) for acute lower appendage musculoskeletal injuries in collegiate players 365 days post-concussion with high re-injury chances of 1.64 times. Similarly, Brooks et al.'s (2016) prospective cohort study predicted elevated odds of 2.48 times for concussed collegiate players than the control group during a 90 day follow up. Further, Herman et al.'s (2017) prospective cohort study revealed high incidence rates for lower appendage musculoskeletal injuries among exposed concussed and non-concussed athletes (3.39, odds of $50 \%$ ) than the non-exposed group (20\%). Female players reported lower odds ratio (2.75) for lower appendage musculoskeletal injuries compared to male players (3.72). Thus, injury risk has a contextual-orientation to sport type and nature. Handball, by virtue of its evasiveness and $2^{\text {nd }}$ placement in world rankings in terms of speed, puts players at high susceptibility levels to multiple injuries.

Research indicates a correlational existence between neuromuscular control and a player's functionality (Kuenze et al., 2015). Further, determined alterations in neurocognition increase the vulnerability to muscular ruptures (Herman et al., 2017). Incapacity to process environmental cues, creating appropriate neuromuscular response for safe movements, has been reported for players with low neurocognitive routines which amplifies chances of overwhelming quality of mitochondrial motor tenacity (Herman et al., 2017). Reliance on 
precise sensory information usage can subvert musculoskeletal injuries and subsequently stabilize joint movements during dynamic manoeuvres (Nagai et al., 2018). Coupled with aberrant movement patterns and personal risk of injury, athletes become more prone to musculoskeletal injuries (Kiesel, Butler and Plisky, 2014; Haines, Baker and Donaldson, 2014). Thus, unequal force production in musculotendinous units could offset the body's capabilities to proficiently engage into a well-coordinated skilled act due to poor perceptual inefficiencies during motoric engagements eventually causing injury.

Continuous muscular activity has also been linked with different musculoskeletal injuries in athletes. Extreme motion of the posterior foot in the frontal plane, especially a lateral heel strike with undue compensatory pronation causes a "whipping action" on the Achilles tendon that pre-disposes it to tendinoparthy (Longo, Ronga and Maffuli, 2017) an injury often linked to sprints and side cutting manoeuvres in handballers. Besides, multi-factorial running-related injuries often develop but substantially vary in relation to training characteristics, running mechanics and runner's anatomy (Malisoux, Nielson, Urhausen, et al., 2015). The weight-bearing structures and muscles may fail to sustain body weight in such repetitive movements. Vertical ground reactional force externally stresses the body (Davis, Bowser and Mullineux, 2016) during handball running actions. Van der Worp et al.'s (2016) meta-analysis study revealed higher loading rates for vertical ground reactional force tendencies among patients (athletes) with stress fracture history. Higher impact-related variables were reported to risk body and soft tissue injuries (Davis et al., 2016). This could incapacitate the body's ability to absorb shocks allowing force to be transmitted through the bone or tissues causing lower appendage musculoskeletal tissue raptures and damage of the metatarsals and phalanges (mertatasalgia injury).

Figure 1, 2, 3 and 4 show common vulnerable injury sites and related structures of the lower appendages susceptible to injuries during participation.

The lateral stabilizers are critical (figure 1 ) in static stabilization of the knee during handball knee biomechanics (Hughes, 2018; Naraghi and White, 2016). Dynamic and explosive forces during knee extension and flexion manoeuvres may overpower the anterolateral and posterolateral structures' capacities to withstand upper body weight eventually causing chronic and acute multiligamentous posterolateral corner injuries (Beutler and Fields, 2018; Hughes, 2018; Rastogi et al., 2015).

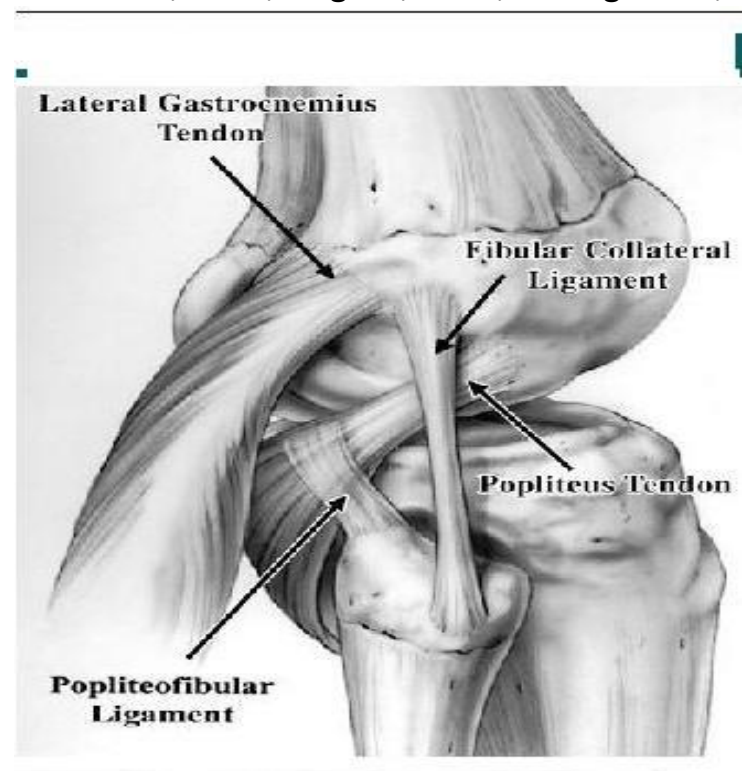

Figure 1: Major Posterolateral stabilizers of the knee vulnerable during dynamic manoeuvres in handball. Source: Naraghi and White, (2016).

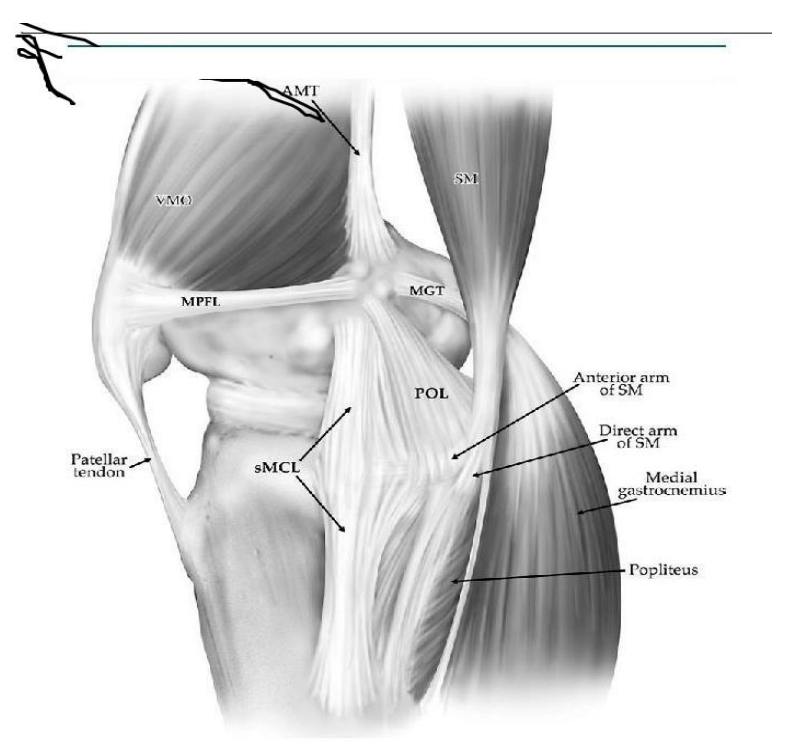

Figure 2: Key medial and posteromedial stabilizers of the knee. Source: Naraghi and White, (2016)

For instance, quadriceps tendinopathy, articular cartilage injury, tendon dislocation, osteoarthritis, varus and valgus knee displacements can surface up (Beutler and Fields, 2018). Non-operative treatment of posterolateral corner injuries requires knee 
immobilization in full extension with weight bearing protection for 2 weeks followed by progressive functional rehabilitation of quadriceps strengthening with return-to-sport in 8 weeks. Postoperatively, the injured site would need a 4 week immobilization with protected weight bearing through casts to control leg external rotation (Hughes, 2018).

Biomechanically, the role of the anterolateral capsule is to bring under control interior tibial rotary motion (Volker, Herbst, Burnham and Fu, 2018). Rupturing of the anterolateral knee structures causes rotary knee insecurity which is related to $A C L$ tears (Musahil et al., 2017) commonly found in handball due to its sudden directional changes, sidecutting and pivoting manoeuvres. Eventually, chronic overuse injuries and knee osteoarthritis can develop causing chopart complex injuries subsequently affecting multiple structures (Halah, Stern and Drittenbass, 2017) especially in lower body appendages through high knee force production levels. This causes long-lasting inflammation, tenderness and disability and post traumatic flat foot deformity (Halah et al., 2017).

The Medial Collateral Ligament provides firmness to valgus strength during full Range of Motion of the knee including during exterior and interior rotation of the tibia. The Postero Oblique Ligament restrains internal rotation and posterior transformation of the tibia during knee extension and flexion (Petersen et al. in Naraghi and White, 2016). Poor knee biomechanics can result in varus and valgus knee displacements to the posteromedial structures (Naraghi \& White, 2016) leading to patellar tendinopathy, patellar dislocation and adductor tendon tears (Beutler and White, 2018). Acquaintance of multi-dimensional risks regarding injury cause critically assists sports medicine clinicians so that correct biomechanical movements are stressed to reduce injury risk.

Figure 3 and 4 depict that the kind of movement patterns during participation has resonant impact on the oesteo-articular devices of the foot and ankle often with distressful effects (D'Hooghe., 2018). Ground reactional forces, varus and valgus forces together with asymmetrical movement patterns have often led to Achilles tendonitis, Anterotalofibular injuries, Posterotalofibular injuries, Calcaneofibular injuries and ankle sprains/strains (Rastogi et al., 2015). Monaco et al.'s (2019) 2 year prospective cohort study among 164 Spanish league male handball players (12-27 years)
(2011-2013), indicated that ankle sprains and cartilage injuries represented $18 \%$ of total injuries incurred. 'Ligamental raptures (52\%), joint sprains and muscle strains (33\%) were reported to be common during match and training'.

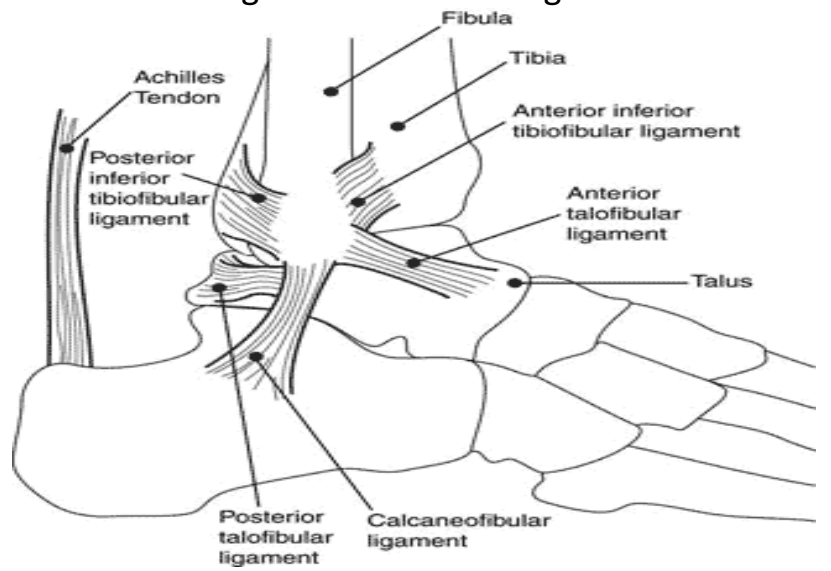

Figure 3: Lateral View of Ankle Structures. Retrieved from (http://www.niams.nih.qov Online Jan 2019)

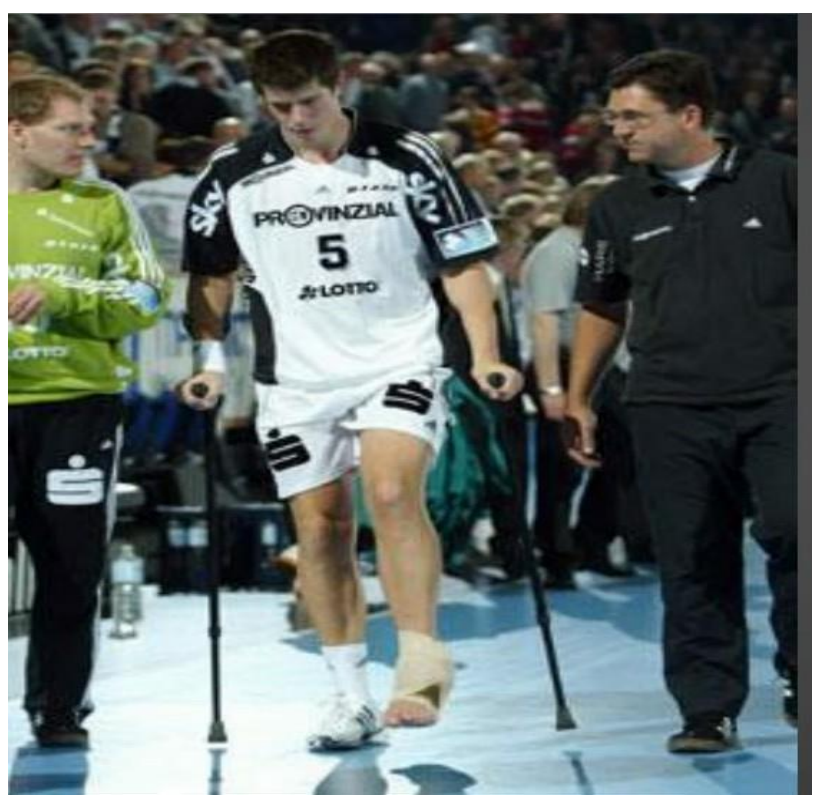

Figure 4: Ankle injuries-common disability feature in handball. Source: Dr. Laver, (2016) Orthopaedics \& Sports Medicine Department, Tel Aviv University Hospital

Similarly, Achenbach et al.'s (2017) parallel study reported $22 \%$ ankle injuries for the intervention group and $25 \%$ for the control group among 279 adolescent male and female Germany handball league. Ankle sprains had $20 \%$ while a score of $22 \%$ was reported from the control group. Ankle injuries accounted for the highest incidence rate. Generally, sprains had 32\% (intervention group), 34\% (control group); strains 12\%, 3\%; dislocations 4\%, 3\%; fractures $6 \%, 9 \%$ and concussion $9 \%$. This suggests undue pressure on sports medicine professionals and resources in re-instating players back to pre- 
injury conditions despite variations in injury severity, restorative patterns and adherence levels during transitional phases of rehabilitation. As noted by Solanki and Solanki (2017), sports medicine professionals should enticingly provide basis for core-profiling their athletes' health status. Subsequently, the essence of inner order and harmony between body and whole cosmos comes in handy if athletes' physiological, psychological, emotional and social tenets are to be fully resolved (Ren, 2019; Pisk, 2017).

\section{Research Methodology}

This section presents the methodology that guided the study.

\section{Research Design}

This study employed a prospective cohort design based on a quantitative approach and informed by a Positivist Philosophy of Positivism. From a Positivist view point, the reality and truth regarding sports injury risks for this study were prospectively derived, measured and quantified (Basit, 2010; Lincolin and Guba, 1985) from injured and nonplayers' patterns of exposure in order to draw up outcome-based conclusions.

\section{Population and sampling}

Participants for the study were drawn from team's technocrats (coaches, physiotherapists, psychologists, handball players and fitness trainers) from 13 Teachers' Colleges in Zimbabwe. Male and female players were from original teams for the entire period of study. Stratified random sampling was adopted in drawing a systematic sample size of 228 respondents from each group (Magwa and Magwa, 2015; Cohen et al., 2007). Yamani's (1970) formula was then used to determine a manageable sample size (Collins et al., 2017) while Bowley's proportional allocation formula statistically distributed respondents into their respective strata.

\section{Instruments used}

A questionnaire with closed-ended items was used to collect 'real time data' (Bailasha et al., 2015) in respect of the study design.

\section{Validity and Reliability}

In checking for the instruments' quality, Cronbach alpha statistic was used to calculate the internal consistence of the questionnaire items. As averred by Taber and Kith, (2018), a critical value of 0.7 or above is indicative of acceptable reliability of an instrument's internal consistency. This study's instrument yielded a reliability score of 08 . Its validity was then pre-tried using a pilot study of one handball team's technical staff and players prior to its administration to the intended sample (Maheshurani, 2017).

\section{Statistical Treatment of Data}

Data was quantitatively presented and analysed using IBM SPSS Statistics Version 23 and presented on a multi-part graph.

\section{Ethical considerations}

Ethical clearance was sought from the Zimbabwe Open University Higher Degrees Committee, Ministry of Higher and Tertiary Education, Science and Technology Development and Teachers' College authorities. Informed consent was sought from study participants drawn from research sites where the study was conducted. The principals of confidentiality and anonymity regarding collected data were highly ingrained as important features during the study.

\section{Results and Discussion}

In this section major findings based on risks connected with active involvement in sport and injury management protocols are explicated. The extent of sports medicine professionals' conversance regarding risks pre-disposing players to unsafe playing environments are presented. The section then unfolds into a systematic discussion and ends up providing recommendations that could significantly trim down the burden of injury risks during training and competitive contexts.

Coaches and fitness trainers' support synergy levels on injured players is summarized using statements measured on the Likert scale. The data yielded in this study indicated that the majority of respondents (38\%) sometimes discuss players' return to sport contexts with the team physiotherapist. While significant proportions highlighted that they sometimes (32\%), always $(28 \%)$ or often $(16 \%)$ let player progress sport participation upon injury sustenance, more than $20 \%$ reported that they never did so. Despite this low awareness level, surprisingly, the bulk of respondents (65\%) seem to regularly pay visits to injured players, a critical psychotherapy measure, yet players' health integrities should be highly honored than protecting institutional or individual sporting turf. Despite high proportions of respondents (60\%) who never recalled recuperating players for crucial matches, nevertheless, a significant number (40\%) confirmed they sometimes or often requested players to report for such 
matches. Thus far, coaches and trainers may sacrifice players' health in order to win important matches though the majority advised that they sometimes, often or always ask players to stop playing $(84 \%)$ or never do so $(16 \%)$ to injured players. This surprising development is indicative of non-existence and non-adherence to rehabilitation protocols by health service providers. High load intensities of this nature are likely to overwhelm recuperating musculoskeletal tissues capacities eventually re-igniting their index injuries.

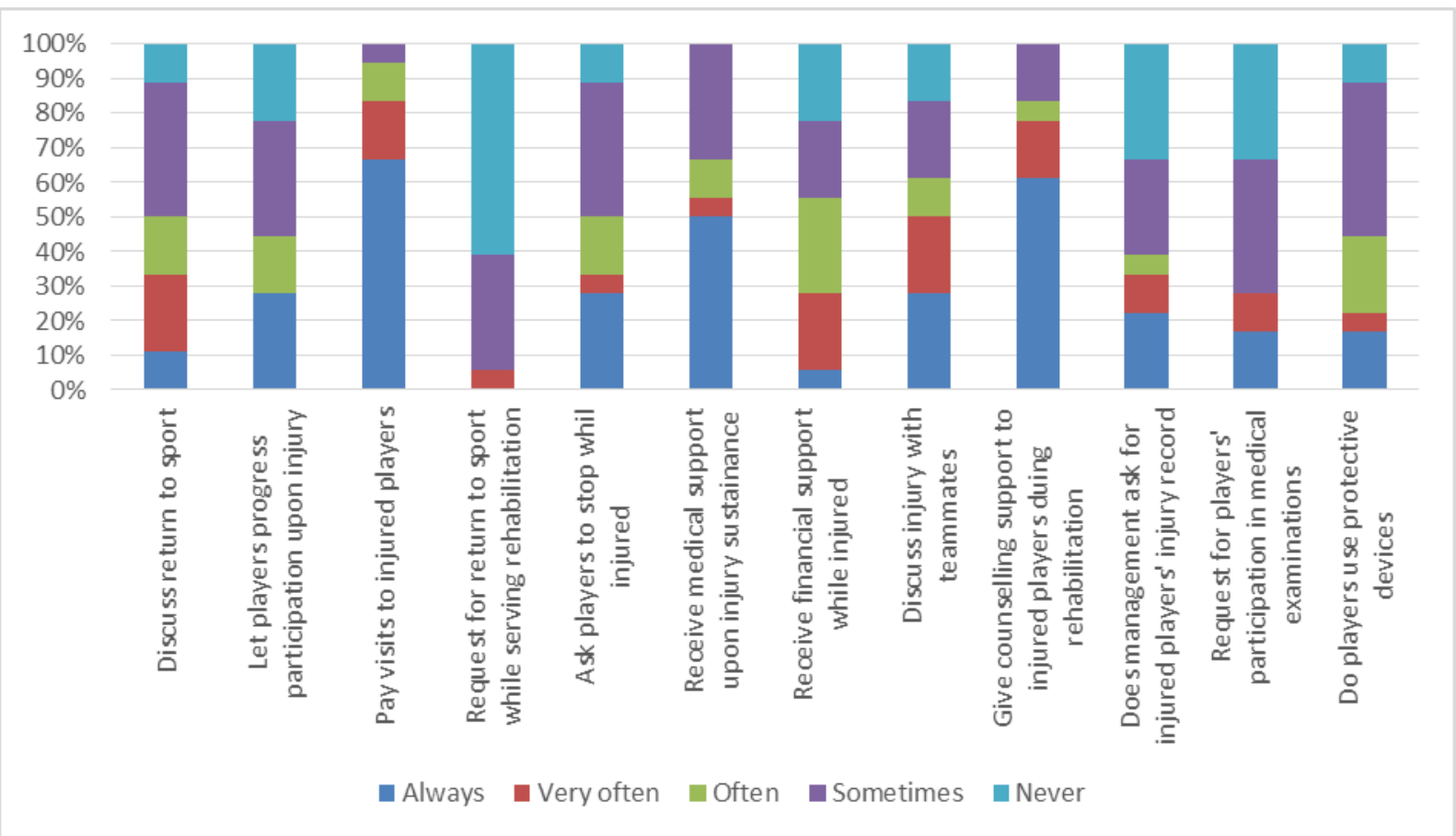

Figure 5: Support synergy inventory rendered by coaches and fitness trainers to injured players

Results suggest that all players received medical support to different extents as evidenced by significant proportions that always (50\%) or sometimes (38\%) got medical support while out injured. Accordingly, it appears that there is awareness on the importance of providing medical support to an injured player. A large section received financial support (61\%) while recuperating from injury, to different levels. However, over a fifth $(21 \%)$ indicated that injured players never or sometimes $(17 \%)$ receive financial assistance from their institutions while undergoing injury rehabilitation. Most coaches and trainers highlighted that they discuss players' injuries with teammates $(60 \%)$ with the rest indicating that they never $(17 \%)$ or sometimes $(21 \%)$ shared these predicaments with teammates. A large section of respondents (82\%) affirmed availing counselling sessions to players during rehabilitation with (18\%) confirming infrequent exercises of this nature. Although a smaller segment confirmed scarce knowledge, results suggest high awareness levels of sport injury management.
Shortway et al. s' (2017) study reported that $27 \%$ of 215 fitness trainers never ever referred athletes for counselling services prior to active participation. Results reinforce this study's figure of $18 \%$. The $82 \%$ affirmation in this study is above Clement et al. s' (2015) figure of $70 \%$ endorsement from fitness trainers, psychologists and councillors' involvement in the psychosomatic and physiological treatment of athletes. Despite these differences, a further introspection of this study's figures, makes it more distinct, that the injured athlete's full functionality is being addressed by a collaborative care team of sports medicine professionals as reported in previous studies (Rahman, 2017, Pabian et al., 2017; Lisbon et al., 2016; Speerin et al., 2014). Astonishingly, a large section of respondents never asked for injured players' injury records, pre participatory medical examinations (64\%) or sometimes $(70 \%)$ carried out these exercises, suggesting poor awareness on the significance of past records in managing and protecting players' health status. Yet medical records and screening examinations critically serves as informational support in enlightening therapists and coaches on 
players' undetected impairments, activity limitations and participation restrictions so that appropriate loadings can be planned and instituted based on individual limits (Sullivan et al., .2019; Targett et al., 2018). This implies an existing burden service gap and awareness deficiencies on sports injury risk management and care settings among sports medicine professionals in tertiary handball.

Allotment of results further envisage low levels regarding the criticality of health check examinations in injury management as suggested by the high percentage of respondents who never or sometimes $(70 \%)$ requested injured players to be medically examined. Consequently, high reports of injury cases and opportunity costs due to injury risks are inevitable. Another important finding emerging from the results is that only a few players always use protective devices (16\%). In contrary, over $12 \%$ reported that players never use devices to prevent injuries. This is indicative of lack of understanding on risk factors or financial constraints to avail protective devices for players. In effect, the greater segment of players sometimes (44\%) or often/very often $(28 \%)$ used protective devices indicating awareness on the critical value of safety as risk management practices for injury reduction compared to a few who lack this basic understanding.

Basing on the results of this study, sports medicine professionals, by virtue of their proximity as front line health team involved in resolving players dented physiological, psychological, emotional and social dimensions require basic understanding on the risks, grounds and related aftermaths of injuries. Sports injury prevention need to find its pathways through early detection of possible volatile exposures pre-disposing players to injury. Well formulated guidelines and skill sets should insulate their code of ethics that clearly delineates their roles within the precincts of athlete-centeredmedicine. Enabling of players' hope pathways need to be developed through encouraging restorative settings that takes into cognizance players' well being and health integrities as critical basis upon which safe participation turfs in sport are founded. Thus, as averred by Ren et al. (2019) and Pisk (2017) the essence of inner order and harmony between body and whole cosmos (social, emotional mental and physiological restoration) significantly provides resonant core-profiling basis (Solanki \& Solanki, 2017 ) in athletes. Hence this could serve in reducing high opportunity risks and costs in injury-prone environments.

Emerging findings from this study revealed that the issue of health-check on players' physiological status was not highly prioritized yet periodic medical examinations serves to enlighten sports clinicians with defects that might need attention prior to reentering into competitive bouts. A significant section of coaches and fitness trainers risked players for important matches when they were still serving their rehabilitation routes, a clear testimony of failure to honor players' health integrities in view of personal or institutional satisfaction.

\section{Conclusions and Recommendations}

This section provides the conclusions and then gives the recommendations.

\section{Conclusion}

Sports medicine professionals lacked skill set expertise regarding critical informational sources of players' objective profiles, injury records, and preparticipation medical screenings as evidence-based practices for addressing physiological defects and risks upon return-to-sport contexts. Well formulated guideline-inclined preventive injury risk protocols with player-centered medicine practices were lowly esteemed. Furthermore, the issue of regularized inservice professional development clinics lacked.

\section{Recommendations}

The study recommends that sports medicine professionals should seriously consider players' objective injury records, profiles and preparticipation medical examination practices as critical informational sources so that all physiological defects are fully resolved upon their return-to competitive contexts. Further, establishment of well formulated guideline-inclined preventive injury risk management protocols resonating player-centered medicine approaches could effectively trim down epidemiologic opportunity injury risks and costs and improve the critical base for players' odds of participation. More importantly, regularized in-service professional development trainings for sports medicine professionals through symposiums should inexorably be an esteemed epitomic pedestal delved towards improving expert base levels in view of the ever-changing preventive and risks management dynamics involved in sports participation. 


\section{Reference}

Achenbach, L., Krutsch, V., Weber, J., Nelich, M., Luig, P., Loose, O., Angele, P., \& Krutsch, W. (2017). Neuromuscular exercises prevent severe knee injury in adolescent team handball players. European Society of Sports Traumatology, Knee Surgery, Arthroscopy, Doi. 10.1007.

Achenbach, L., Krutsch, V., Weber, J., Nelich, M., Luig, P., Loose, O., Angele, P., \& Krutsch, W. (2017). Neuromuscular exercises prevent severe knee injury in adolescent team handball players. European Society of Sports Traumatology, Knee Surgery, Arthroscopy, Doi. 10.1007.

Atchison, B., Ruston, A., Martin, P., Soundy, A., and Heneghan, M. R. (2020). British Medical Journal, 10, (11), eo38214.

Australian Standard, (2004). Injury Risks in Sport, Australia, Routledge Publishing.

Bahr, R., Clarsen, B., et al., (2020). International Olympic Committee Consensus Statement: Methods for Recording and Reporting of Epidemiological Data on Injury and IIIness in Sports, Orthopaedic Journal of Sports Medicine, 8(2), 2325967120902908,2020.

Baar, K. (2017). Minimising Injury and Maximising Return to Play: Lessons from Engineered Ligaments. Sports Medicine, 47 (1), 5-11.

Bailasha, N, K., Kibera, L, K., Rintaugu, E, G., and Mwisukha, A, (2015). Assessment patterns of sports injuries in Selected Ball games during a season of the Kenyan National League, Journal of Health and Science, 4 (2), 34-40.

Basit, T, N. (2010). Conducting research in educational contexts, Bloomsbury Publishers. New York.

Battagia, A., Kerr, G., and Tamminen, K. (2021). A Grounded Theory of the Influences Affecting Youth Sport Experiences and Withdrawal Patterns, Journal of Applied Sport Psychology, 1-41, 2021.

Beutler, A., and Fields, K, B. (2018). Approach to the adult with knee pain likely of musculoskeletal origin, British Journal of Sports Medicine, 30, 76-80.
Berber, U., and Mollaogullan, H. (2020). The effect of service quality on satisfaction of athletes participating in sport programmes, European Journal of Physical Education and Sports Science, 6 (1), 2020. http://dx.doi.org/10.46827/ejpe.44i02802.

Breivik, G., Johnson, J. H., and Augustad, T. (1994). In Chen, R., Baluch, B., and Duffy, L. (2018). Defining Extreme Sport: Conceptions and Misconceptions: Frontiers in Psychology, https://doi.org/10.3389.

Brooks, M. A. (2016). Concussion increases odds of sustaining a lower extremity injury after Return to Play among collegiate athletes. American Journal of Sports Medicine, 44 (3), 742-747.

Broglie, S. P., Suma, T., and Ashton-Miller, J. A. (2014). High school and collegiate football athletic concussions: A biomechanical review. Annals of Biomedical Engineering, 40, 37-46.

Chiu, S. L., Ostering, L., and Chou, L.S. (2016). Concussion induces a gait inter-joint coordination variability under conditions of divided attention and obstacle crossing. Gait Posture. British Journal of Sports Medicine, Pubmed.

Clement, j., Brunet, J., and Davis, I, S. (2015), Rehabilitation following sports injury, British Journal of Sports Medicine, 9 (6), 93-98.

Cohen, S., Diverts, D., and Miller, G, E. (2007). Pedagogical Stress and Disease, American Medical Association, 298 (14), 1685-1687.

Collins, G, H., Davis, I, S., Chou, L, S. (2017). Rehabilitation of Sports Injuries, Journal of Sport and Science, 12 (5), 37-45.

Davis, I. S., Bowser, B. J., and Mullineux, D. R. (2016). Greater vertical impact loading in female runner with medically diagnosed injuries: a prospective investigation. British Journal of Sports Medicine, 50.Doi.1011 36/bj sports-2015-094579.

D'Hooghe, P., Kaux, J. F., Bukva, B., Abdellatif, N., Pereira, H., Camont, M., and Karlsson, J. (2018). Foot and Ankle problems in Handball. Handball Sports Medicine, pp.341354. 
Dore, I., Sabiston, E.M., Sylvester, P., Brunet, J., and Belanger, M. (2019). Years Participating in Sports During Childhood Predicts Mental Health in Adolescence: A 5 year Longitudinal Study, Journal of Adolescent Health, 64 (6), 790-796.

Fordsdyke, D. (2021). Are you a Buffer or An Amplifier? The role of Social Support in the effective Return-To-Sport Following Injury, From Risk to Retirement, The Psychology of Sports Injury, 8 (6),107-116.

Frontera, W, R. (2003). Epidemiology of sports injuries: Implications for Rehabilitation, https://doi.org/9780470757178.ch1.

Fuller, C, W., Dvorak, A., and Junge, A. (2006). Application of Risk Management in Sport, Sport Medicine, 34, (6), 349-352.

Gouttebarge, V., Bindra, A., Blauvelt, C., et al., (2021). International Olympic Committee (IOC) Sport Mental Health Assessment Tool 1 and Sports Mental Health Recognition Tool 1: Towards better support of athletes mental health, British Journal of Sports Medicine, 55 (1), 30-37.

Grinden, H., Granon, L. P. Risberg, M.A. et al. (2017). How does a combined pre-operative and post-operative rehabilitation program influence the outcome of Anterior Cruciate Ligament reconstruction 2 years after injury? A comparison between patients in the Delware-Oslo $\mathrm{ACL}$ cohort and the Norwegian National Knee Ligament Registry. British Journal of Sports Medicine, 49 (6), 385-389.

Guba, E, G., and Lincolin, Y, S. (1985). Establishing Trustworthiness, Naturalistic inquiry, 289 (331), 289-327.

Halah-Kutaish, R. S., Stern, L., and Drittenbass, M. A. (2017). Injuries to the chopart joint complex: A current review. European Journal of Orthopaedic Surgeon and Traumatology, 27 (4), 425-431.

Herman, D., Jones, D., Harrison, A., Moser, M., Tillman, S., Farmer, K., Pass, A., Clugson,J., Hernandez, J., and Chmielewski, S. (2017). Concussion increase te risk of subsequent lower extremity musculoskeletal injury in collegiate athletes. Sports Medicine, 47 (5), 1003-1010.
Herring, S, A., and Kibler, W, B. (1998). A framework for Rehabilitation, Functional Rehabilitation of sports and musculoskeletal injuries, Maryland, Aspen Publishers.

Hickey, D., Solvig, V., Cavalhen, V., Harold, M. and McKenna, L. (2017). Scapular dykinesis increases the risk of future shoulder pain by 43\% in asymptomatic athletes: a systematic review and meta-analysis. British Journal of Sports Medicine, 1-10.

Hughes, M. (2018). Posterolateral Corner Injury. Image Medical. Inc. Knee and Sports Orthopaedics, 29, 47-68.

Hulme, A., and Finch, C. F. (2015). From monocausality to systems thinking: a complementary and alternative conceptual approach for better understanding the development and prevention of sports injury. Injury Epidemiology, 2(31), 1-17.

Kam-Ming Mok (2015). Reliability and methodical concerns of vertical drop jumping and side step cutting tasks. Implications for Anterior Cruciate Ligament Injury risk screening. Dissertation from the Norwegian School of Sport Science.

Kiesel, K., Butler, R., and Plisky, P. (2014). Prediction of injury by limited and asymmetrical fundamental movement patterns in American football players. Journal Sports Rehabilitation, 23 (2), 88-94.

Kuenze, C, M., Foot, N., Saliba, S, A., and Hart, J, M. (2015), Drop-Landing Performance and knee extension Strength After Anterior Cruciate Ligament Reconstruction, Journal of Athletics Training, 50 (6), 596-602.

Landreau, P., Zumstein, M. A., Lubiatouski, P., and Lavern, L. (2018). Shoulder Injuries in Handball. Handball Sports Medicine, 29, 125-146.

Laver, D. (2016). Orthopaedics and Sports Medicine Department, Tel Aviv University Hospital.

Lisbon, D., Allin, D., Cleck, C et al., (2016). Improved knowledge, attitudes and behaviours after implementing of Team STEPPS training in an academic emergency department: a pilot study, American Journal of Sports Medicine, 8 (10), 83-97. 
Longo, U. G., Ronga, M., and Maffuli, N. (2017). Achilles Tendinoparthy. Aetiology and management. Journal of Sports Medicine, https//www.researchgate.net/publication.

Lynall, R, C., Kerr, Z, Y., Djoko, A., et al., (2016). Epidemiology of National Collegiate Athletic Association men's and women's tennis injuries, 2014-2015, British Journal Of Sports Medicine, 50 (19), 132-140.

Magwa, S and Magwa, W. (2015). A Guide To Conducting Research: A Student's Handbook, Singapore, Strategic Book Publishers, Palmer.

Maheshurani, V. (2017). Fundamental Concepts of Research Methodology, India, Roorke.

Majewski, R. (2018). Adult-and peer-created motivational climates in sport and injury rehabilitation. Electronic Thesis and Dissertations, University of Northern lowa.

Malisoux, L., Nielson, R, O., and Urhausen, G. (2015). $A$ step towards understanding the mechanics of running-related injuries, Journal of Science and Medicine in Sport, 18, 523-528.

Martini, D, N., Sabin, M, J., DePesa et al., (2011). The chronic effects of concussion gait, Archives of Physical Medicine and Rehabilitation, 92 (4), 585-589.

Monaco, M., Rincon, J.A.G., Ronsano, B. J. M., Whitely, R., Lopez, F. S., and Rodas, G. (2019). Injury incidence and injury patterns by category, player position and maturation in elite male handball players. Biology Sports, 36 (1), 67-74.

Musahil, V., Getgood, A., Neyret, P., Claes, S., and Burnham, J. M. (2017). Contributions of the anterolateral complex and the anterolateral ligament to rotary knee stability in the setting of $\mathrm{ACL}$ injury: a round table discussion.

Nagai, T., Schilaty, N. D., Strauss, J. D., Crowley, E. M., \& Hewett, T.E. (2018). Analysis of Lower Extremity Proprioception for ACL Prevention; Current Opinion. Sports Medicine, 48 (6), 1303-1309.

Naraghi, A. M., \& White, L. M. (2016). Imaging of Athletic Injuries of knee ligaments and menisci: Sports Imaging Series. Radiology, 281, 1-18.
Nemcek, D. (2017). Subjective Quality of Life Analysis in Population Regularly Participating in Sport with Different Health Status, International Journal of Arts Humanities and Social Sciences, 2 (7), 25-33.

Pabian, P, S., Dyson, J., and Levine, C. (2017). Physical Therapist Productivity using a Collaborative Clinical Education Module Within an Acute Care Setting,: A Longitudinal Study, Journal of Physical Therapy Education, 31 (2), 11-17.

Palmer, C. (2002). In Cohen, R., Baluch, B., \& Duffy, L. (2018). Defining Extreme Sport: Conception and Misconceptions. Frontiers in Psychology. https://doi.org/10.3389.

Pierpoint, L, A., and Collins, C. (2021). Epidemiology of Sports-Related Concussion, Clinics in Sports Medicine, 40 (1), 1-18.

Pisk, J. (2017). Wisdom of the body in Sport and Exercise Practices: Physical Culture and Sports Studies and Research, Volume LXXV, 15-22, Doi.10.1515/pcssr.

Ren, Y., Gui, J., and Chen, Y. (2019). The clue of Art: A Philosophical Interpretation of Ancient Greek Sport from the Philosophy of art, Argos, 36 (75), 244-253.

Rastogi, A., Whelan, D., Martin, R., Mak, W., Pearce, D., Toronto, C. A. and Calgary. C. A. (2015). Multi-ligamentous knee injuries-MRI injury patterns at a glance. European Society of Musculoskeletal Radiology, www.essr.org.

Rahman, h. (2017). Concept of athlete-centred care, International Journal of Health Sciences, 11 (7), 73-80.

Shahri, M, H. M., Ghafouri, F., Honeri, H., and Shahlee, J, 2019). Designing the mental health pattern of athletes participating in educational sport, Research Paper, Journal of Research on Educational Sport, 9 (16), 1738.

Sanderson, F. H. (2021). Analysis of Anxiety Levels in Sport Taylor and Francis Group.

Shen, W., Junzhou, Y., Qian, Y., and Junyi, Z. (2019). Implementation intensions improve selfefficacy and behaviour regardless of task difficulty, Social, Behaviour and Personality' Scientific International Journal Publishers, 47 (4) https://doi.org/10.2224. 
Shortway, K, M., Wolanin, A., Webster, K, E., et al., (2017). Acceptance and commitment therapy for injured athletes: Development and preliminary feasibility of the return to action protocol, British Journal of Sports Medicine, 10 (6), 23-28.

Solanki, V and Solanki, J. (2017). Potential contribution of Physical Education tin achieving the aims of Education, International of Physiology, Nutrition and Physical Education, 2 (2), 243-248.

Speerin, R., Slater, H., Li, L., Moore, K., et al., (2014). Moving from evidence to practice models of care for the prevention and management of musculoskeletal conditions, Best Practice and Research, Clinical Rheumatology, 28 (3), 479-515.

Sullivan, S, B, O., Schmitz, T, J., and Fulk, G. (2019), Physical Rehabilitation, $9^{\text {th }}$ Ed, Davis Company, USA.

Taber, K, S., and Keith, J. (2018). The use of Cronbach's alpha when developing and reporting research instruments in science education, Research in Science Education, 48, 1273-1296.
Targett, S., Bere, T., and Bahr, R. (2018). The role of pre-participation assessment (PPA) and screening in handball, Handball Sports Medicine, 115-124.

Trompeter, K., Fett, D., and Platen, P. (2017). Prevalence of Back Pain in Sports: A systematic review of the literature, Sports Medicine. 47 (6), 1183-1207.

Van der Worp, H., Vrielink, J. W., and Bredeweg, S. W. (2016). Do runners who suffer injuries have higher vertical ground reaction forces than those who remain injury free? A systematic review and meta-analysis. British Journal of Sports Medicine, 50, 450-457.

Volker, M., Herbst, E., Burnham, J. M., and Fu, F. H. (2018). The Anterolateral Complex and Anterolateral Ligament of the Knee. Journal of American Academy of Orthopaedic Surgeons. 26 (8), 261-267.

Yamani, T. (1970). Statistics: Introductory Analysis, New York, Harper and Row Publishershttps: //w ww.niamas.nih.gov Online Jan 2019. 